\title{
Seismic ray tracing in anisotropic media: A modified Newton algorithm for solving highly nonlinear systems
}

\author{
Yanghua Wang ${ }^{1}$
}

\begin{abstract}
Seismic ray tracing with a path-bending method leads to a nonlinear system that has much stronger nonlinearity in anisotropic media than the counterpart in isotropic media. Any path perturbation causes changes to directional velocities, which depend not only upon the spatial position but also upon the local propagation direction in anisotropic media. To combat the high nonlinearity of the problem, the Newton-type iterative algorithm is modified by enforcing Fermat's minimum-time principle as a constraint for the solution update, instead of conventional error minimization in the nonlinear system. As the algebraic problem is incorporated with the physical principle, it is able to stabilize the solution for such a highly nonlinear problem as ray tracing in realistically complicated anisotropic media. With this modified algorithm, two ray-tracing schemes are presented. The first scheme involves newly derived raypath equations, which are approximate for anisotropic media but the minimum-time constraint will ensure that the solution steadily converges to the true solution. The second scheme is based on the minimal variation principle. It is more efficient than the first one as it solves a tridiagonal system and does not need to compute the Jacobian and its inverse in each iteration. Even in this second scheme, Fermat's minimum-time constraint is employed for the solution update, so as to guarantee a robust convergence of the iterative solution in anisotropic media.
\end{abstract}

\section{INTRODUCTION}

Seismic raypath is a trajectory along which wave energy propagates, and the group velocity determines the speed of signal propagation and energy transport along the raypath (Červený, 2001). In isotropic media, the raypath and the group velocity vector coincide with direction normal to the wavefront (Julian and Gubblins, 1977; Pereyra et al., 1980; Červený, 2001; Rawlinson et al., 2007). In anisotropic media, ray tracing remains a challenging problem especially in terms of numerical calculation, although the fundamental theory of anisotropy is well-known (Crampin, 1981; Fryer and Frazer, 1984; Shearer and Chapman, 1988; Červený, 2001; Wang, 2013). The raypath and the group velocity vector are not perpendicular to the wavefront. As the group velocity vector is parallel to the energy flux, an exact expression for the group velocity vector in terms of the group angle is difficult to obtain and is too cumbersome for practical use. This paper attempts to derive ray-tracing equations presented in terms of the group velocity. With a proper constraint, these equations are applicable to ray tracing in anisotropic media.

For ray tracing in anisotropic media, the Hamilton equationbased method has a compact and elegant mathematical form, in which a Hamiltonian function can be modified to take into account the anisotropic property (Červený, 2002; Vavryčuk, 2006) and even the attenuation property (Červený and Pšenčík, 2005; Vavryčuk, 2008). Perturbation is a pragmatic approach to make a first-order approximation around an existing solution in the neighborhood or in an isotropic background (Červený and Jech, 1982; Hanyga, 1982; Farra and Madariaga, 1987; Jech and Pšenčík, 1989), but is only applicable to weak anisotropic media.

This paper presents path-bending methods for ray tracing between two end points in anisotropic media. Practical implementation of a bending method often leads to a system of nonlinear equations, which can be solved by using a standard Newton-type iterative algorithm (Kelley, 2003). This nonlinear system in anisotropic media has much stronger nonlinearity than the counterpart in isotropic media. In an isotropic case, path perturbation will cause velocity changes and these velocity changes will further affect the raypath. In anisotropic media, any path perturbation will cause changes in directional velocities, and these changes depend not only upon the spatial position but also upon the local propagation direction. Due to high nonlinearity, it has been found that Newton's

\footnotetext{
Manuscript received by the Editor 14 March 2013; revised manuscript received 19 August 2013; published online 5 November 2013.

${ }^{1}$ Imperial College London, Department of Earth Science and Engineering, Centre for Reservoir Geophysics, London, UK. E-mail: yanghua.wang @imperial ac.uk.

(C) 2013 Society of Exploration Geophysicists. All rights reserved.
} 
iterative procedure, which conventionally relies on the minimization of the error in the nonlinear system, will not work for anisotropic cases. To combat the high nonlinearity of the problem, this paper proposes to modify the Newton-type iterative algorithm by enforcing Fermat's minimum-time principle as a constraint for the solution update. As it enforces a physical principle into a solution procedure of nonlinear algebraic equations, it is able to stabilize the iterative procedure, even in complicated anisotropic cases.

In this paper, the modified Newton algorithm is applied to two ray-tracing schemes in anisotropic media. One scheme (scheme 1) is based on raypath equations. Another scheme (scheme 2) is derived from the Fermat's variational principle. In both schemes, a step length for the solution update in the iteration is determined based on Fermat's minimum-time constraint, so as to mitigate potential difficulty due to the high nonlinearity of the problem, to guarantee a steady convergence and to obtain a physically meaningful solution.

\section{MODIFIED NEWTON ALGORITHM}

If a raypath is divided into $n$ segments with mesh points $q_{j}$, for $j=0,1,2, \cdots, n$, discretizing a ray-tracing equation will lead to a system of nonlinear functions,

$$
\mathbf{f}(\mathbf{w})=\mathbf{0},
$$

where $\mathbf{w}$ is a vector of unknown mesh points $q_{j}=\left(x_{j}, z_{j}\right)$, for $j=1,2, \cdots, n-1$, between two end points $q_{0}$ and $q_{n}$. This nonlinear system can be solved by a Newton-type iterative algorithm, as

$$
\mathbf{w}^{(k)}=\mathbf{w}^{(k-1)}-\left[\mathbf{J}\left(\mathbf{w}^{(k-1)}\right)\right]^{-1} \mathbf{f}\left(\mathbf{w}^{(k-1)}\right),
$$

where $\mathbf{J}(\mathbf{w})$ is Jacobian matrix of $\mathbf{f}(\mathbf{w})$ with respect to $\mathbf{w}$, and $k$ is an iteration index.

If coefficients in the nonlinear functions were fixed to constants, it would be a simple mathematical exercise. In seismic ray tracing, path perturbation leads to velocity changes which further affect the raypath. In the isotropic case, this iterative approach might still work, as long as the initial guess is sufficiently close to the final solution. However, such velocity changes in anisotropic media not only depend upon the position of each point along a raypath, but also upon the local propagation direction. It is found that an iterative procedure that relies on the minimization of the errors in nonlinear functions does not work for the anisotropic case because of its much higher nonlinearity than the counterpart in the isotropic case.

To solve this highly nonlinear problem, the Newton-type algorithm is modified with an optimal updating step, as follows

$$
\mathbf{w}^{(k)}=\mathbf{w}^{(k-1)} \pm c^{\beta}\left[\mathbf{J}\left(\mathbf{w}^{(k-1)}\right)\right]^{-1} \mathbf{f}\left(\mathbf{w}^{(k-1)}\right),
$$

where $c$ is a constant $0.1 \leq c<0.5$, and $\beta$ is an integer selected among $\{0,1, \cdots, Q\}$. The constant $c$ is often fixed to a small value, $c=0.1$ for example, so as to have a stable solution through the iteration. The maximum integer $Q$ (for $\beta$ trials) is not that sensitive and is set to be $Q=8$ in the examples shown in this paper.

The modification has two significant features. The first feature is the positive and negative $( \pm)$ signs, as opposed to just a minus, and is important for a steady convergence. This is due to the high nonlinearity of this specific problem, as the nonlinear system $\mathbf{f}(\mathbf{w})=\mathbf{0}$ has coefficients that also depend upon the solution $\mathbf{w}$; this differs from any algebraic nonlinear equation with fixed coefficients. The scheme with \pm signs will prove to be powerful, as it will be able to dig out the solution from any potential local-minimum trap in the iterative procedure.

The second feature is the enforcement of Fermat's minimumtime principle as a constraint in selecting step length $\left( \pm c^{\beta}\right)$ in this specific ray-tracing problem, instead of minimizing the error $\left(\|\mathbf{f}(\mathbf{w})\|^{2}\right)$ of a nonlinear system in any Newton-type algorithm. Although solution-updating direction is guided by $\Delta \mathbf{w}=$ $[\mathbf{J}(\mathbf{w})]^{-1} \mathbf{f}(\mathbf{w})$, a proper step length is selected from a series of trials $\pm 1, \pm c, \pm c^{2}, \cdots, \pm c^{Q}$, one of which has a minimum nonnegative traveltime along the raypath.

The incorporation of these two features, the \pm signed step length plus the minimum time constraint, makes the modified Newton algorithm a neat solution for highly nonlinear problems. It is demonstrated with two path-bending schemes in the following sections. Bending is an appropriate choice for providing a raypath with minimal traveltime, when studying heterogeneous media with potential triplications, such as tomography. Scheme 1 is presented in the time field, $\tau(x, z)$, along a raypath. Scheme 2 is the total traveltime $T$ between two end points, that is $T=\sum \Delta \tau(x, z)$. In selecting $\pm c^{\beta}$ with a minimal traveltime, it is necessary to evaluate $T$ for every trial. Both schemes employ Fermat's minimum time constraint to find a raypath along which the wave energy propagates.

\section{RAY TRACING BASED ON RAYPATH EQUATIONS}

The first ray-tracing scheme is based on newly derived raypath equations. For the traveltime field $\tau(x, z)$ in the 2D space, the phase slowness components may be defined as

$$
\frac{\partial \tau}{\partial x}=\frac{\sin \phi}{v(\phi)}, \quad \frac{\partial \tau}{\partial z}=\frac{\cos \phi}{v(\phi)},
$$

where $v(x, z, \phi)$ is the phase velocity, and $\phi$ is the phase angle (Wang, 2013). This is the eikonal equation, $\operatorname{read}$ as $\nabla \tau \cdot \nabla \tau=$ $1 / v^{2}$. I derive raypath equations by differentiating (4) with respect to a raypath, $r(x, z)$.

Differentiating $\partial \tau / \partial x$ and $\partial \tau / \partial z$ with respect to $r$ leads to

$$
\begin{gathered}
\frac{\partial}{\partial r}\left(\frac{\partial \tau}{\partial x}\right)=\frac{\partial}{\partial x}\left(\frac{\partial \tau}{\partial r}\right)=\frac{\partial}{\partial x}\left(\frac{1}{V(\theta)}\right) \\
\frac{\partial}{\partial r}\left(\frac{\partial \tau}{\partial z}\right)=\frac{\partial}{\partial z}\left(\frac{\partial \tau}{\partial r}\right)=\frac{\partial}{\partial z}\left(\frac{1}{V(\theta)}\right)
\end{gathered}
$$

where $V(x, z, \theta)$ is the group velocity along the raypath $r(x, z)$, and $\theta$ is the ray angle at $(x, z)$. In equation 5 , the following expression is used

$$
\frac{\partial \tau}{\partial r}=\frac{1}{V(\theta)}
$$

Note also that

$$
\frac{\partial \tau}{\partial r}=\frac{\partial \tau}{\partial x} \frac{\partial x}{\partial r}+\frac{\partial \tau}{\partial z} \frac{\partial z}{\partial r}=\frac{\cos (\theta-\phi)}{v(\phi)},
$$


where $\partial \tau / \partial x$ and $\partial \tau / \partial z$ are phase slowness components defined by 4 in terms of the phase velocity $v(\phi)$ and the phase angle $\phi$, and $\partial x / \partial r=\sin \theta$ and $\partial z / \partial r=\cos \theta$, expressed with the ray angle $\theta$. Comparison between equations 6 and 7 lead to the relationship for velocities $V(\theta)$ and $v(\phi)$, as

$$
V(\theta)=\frac{v(\phi)}{\cos (\theta-\phi)} .
$$

To represent the right-hand side of equation 4 in $V(\theta)$ as well, let us make the following approximations

$$
\begin{aligned}
& \frac{\sin \phi}{v(\phi)}=\frac{\sin \theta}{V(\theta)}\left[1-\left(1-\frac{\tan \theta}{\tan \phi}\right) \cos ^{2} \theta\right]^{-1} \approx \frac{\sin \theta}{V(\theta)}, \\
& \frac{\cos \phi}{v(\phi)}=\frac{\cos \theta}{V(\theta)}\left[1-\left(1-\frac{\tan \phi}{\tan \theta}\right) \sin ^{2} \theta\right]^{-1} \approx \frac{\cos \theta}{V(\theta)} .
\end{aligned}
$$

The conditions are $|1-\tan \theta / \tan \phi| \cos ^{2} \theta<1$ and $|1-\tan \phi|$ $\tan \theta \mid \sin ^{2} \theta<1$.

Therefore, differentiating equation 4 produces

$$
\begin{aligned}
& \frac{\partial}{\partial x}\left(\frac{1}{V(\theta)}\right)=\frac{\partial}{\partial r}\left(\frac{1}{V(\theta)} \frac{\partial x}{\partial r}\right), \\
& \frac{\partial}{\partial z}\left(\frac{1}{V(\theta)}\right)=\frac{\partial}{\partial r}\left(\frac{1}{V(\theta)} \frac{\partial z}{\partial r}\right) .
\end{aligned}
$$

These are the general raypath equations expressed in terms of the group velocity. In isotropic media, they are exact as the ray angle $\theta$ is the same as the phase angle $\phi$. In anisotropic media, they are approximate, but the Fermat's minimum-time constraint will ensure the convergence of a raypath toward the true solution.

In numerical implementation where a raypath is divided into $n$ segments, the length of the $j$ th ray segment, $r_{j}$, between mesh points $q_{j-1}$ and $q_{j}$ is approximated by a straight line, and the local directional velocity $V_{j}$ is measured at the center of the segment (Figure 1a). The differentials (in the first equation) are approximated by finite differencing as follows:

$$
\begin{aligned}
& \left.\frac{\partial}{\partial x}\left(\frac{1}{V}\right)\right|_{x_{j}}=-\left.\frac{1}{V^{2}} \frac{\partial V}{\partial x}\right|_{x_{j}} \approx-\frac{1}{2}\left(\frac{V_{j}^{+}-V_{j}^{-}}{V_{j}^{2} \Delta x}+\frac{V_{j+1}^{+}-V_{j+1}^{-}}{V_{j+1}^{2} \Delta x}\right), \\
& \left.\frac{\partial}{\partial r}\left(\frac{1}{V} \frac{\partial x}{\partial r}\right)\right|_{x_{j}} \approx \frac{2}{\Delta r_{j}+\Delta r_{j+1}}\left(\left.\frac{1}{V_{j+1}} \frac{\partial x}{\partial r_{j+1}}\right|_{x_{j}}-\left.\frac{1}{V_{j}} \frac{\partial x}{\partial r_{j}}\right|_{x_{j}}\right),
\end{aligned}
$$

where $V_{j}^{ \pm}$and $V_{j+1}^{ \pm}$are the perturbed directional velocities corresponding to the position perturbations $x_{j} \pm \frac{1}{2} \Delta x$ (Figure 1b), and $\left.\left(\partial x / \partial r_{j}\right)\right|_{x_{j}}$ and $\left.\left(\partial x / \partial r_{j+1}\right)\right|_{x_{j}}$ are ray directions of two adjacent segments hinged at point $q_{j}$. The same approximation forms apply to differentials in the second equation.

There are totally $2 n-2$ nonlinear equations, for solving $2 n-2$ unknown variables $\left\{x_{j}, z_{j}\right\}$, for $j=1,2, \cdots, n-1$. The modified Newton's algorithm 3 is used to solve this nonlinear system iteratively. Because of iterative updating in $x_{j}$ and $z_{j}$, mesh points $q_{j}$ (for $j=1,2, \cdots, n-1)$ are not regularly sampled along the raypath.

\section{RAY TRACING BASED ON MINIMUM VARIATION}

The second ray-tracing scheme is derived from Fermat's variational principle,

$$
\frac{\partial T}{\partial x_{j}}=0, \quad \frac{\partial T}{\partial z_{j}}=0 .
$$

That is, there is a minimal variation in the total traveltime along the entire raypath $\left\{x_{j}, z_{j}\right\}$. It can be presented as a linear tridiagonal system, so as to improve its efficiency. Note that the entire problem is still nonlinear, as setting the system depends upon the current solution in the iteration.

The total traveltime along a raypath between two end points $q_{0}$ and $q_{n}$ can be estimated numerically by

$$
T=\sum_{j=1}^{n} \Delta \tau_{j}=\sum_{j=1}^{n} \frac{r_{j}}{V_{j}},
$$

where $\Delta \tau_{j}$ is the time interval between any two consecutive points $q_{j-1}$ and $q_{j}, r_{j}=\sqrt{\left(x_{j}-x_{j-1}\right)^{2}+\left(z_{j}-z_{j-1}\right)^{2}}$ is its path length, and $V_{j}$ is the local directional velocity.

As $\partial T / \partial x_{j}=\partial \Delta \tau_{j} / \partial x_{j}+\partial \Delta \tau_{j+1} / \partial x_{j}$, the minimum time variation principle (equation 12) leads to the following equations a)

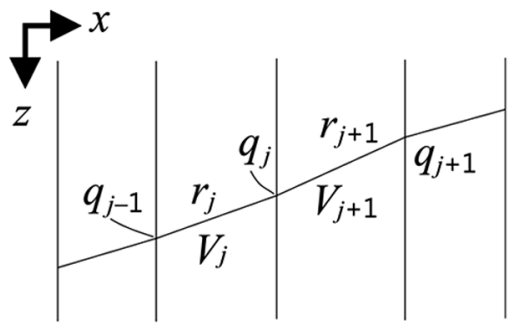

b)

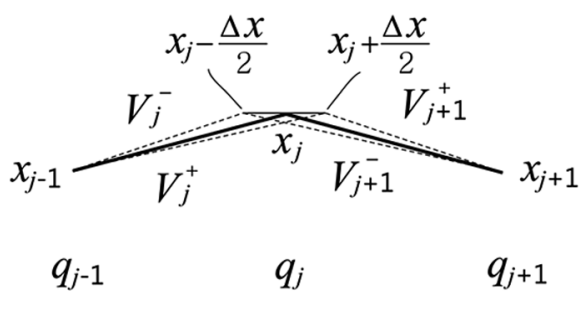

c)

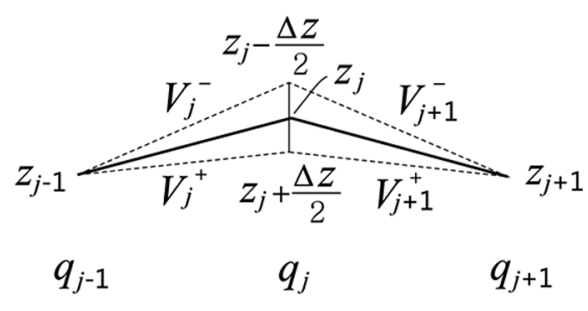

Figure 1. (a) A raypath is approximated by a series of segments with mesh points $q_{j}$. The length $r_{j}$ of the $j$ th ray segment is measured along a straight line, and the local velocity $V_{j}$ is direction dependent. (b) Perturbed directional velocities $V_{j}^{ \pm}$and $V_{j+1}^{ \pm}$corresponding to perturbation $x_{j} \pm \frac{1}{2} \Delta x$ at point $q_{j}$. (c) Perturbed directional velocities $V_{j}^{ \pm}$and $V_{j+1}^{ \pm}$corresponding to perturbation $z_{j} \frac{1}{2}_{2}^{+} \Delta z$ at point $q_{j}$. 


$$
\begin{aligned}
& -\frac{1}{V_{j} r_{j}} x_{j-1}+\left(\frac{1}{V_{j} r_{j}}+\frac{1}{V_{j+1} r_{j+1}}\right) x_{j}-\frac{1}{V_{j+1} r_{j+1}} x_{j+1} \\
& =\frac{r_{j}}{V_{j}^{2}} \frac{\partial V_{j}}{\partial x_{j}}+\frac{r_{j+1}}{V_{j+1}^{2}} \frac{\partial V_{j+1}}{\partial x_{j}}, \\
& -\frac{1}{V_{j} r_{j}} z_{j-1}+\left(\frac{1}{V_{j} r_{j}}+\frac{1}{V_{j+1} r_{j+1}}\right) z_{j}-\frac{1}{V_{j+1} r_{j+1}} z_{j+1} \\
& =\frac{r_{j}}{V_{j}^{2}} \frac{\partial V_{j}}{\partial z_{j}}+\frac{r_{j+1}}{V_{j+1}^{2}} \frac{\partial V_{j+1}}{\partial z_{j}} .
\end{aligned}
$$

On the right-hand side, velocity gradients are estimated numerically. For example, $\partial V_{j} / \partial z_{j} \approx\left(V_{j}^{+}-V_{j}^{-}\right) / \Delta z, \quad \partial V_{j+1} / \partial z_{j} \approx$ $\left(V_{j+1}^{+}-V_{j+1}^{-}\right) / \Delta z$, where $V_{j}^{ \pm}$and $V_{j+1}^{ \pm}$are perturbed directional velocities associated with perturbation $z_{j} \pm \frac{1}{2} \Delta z$ (Figure 1c).

Once the right-hand side quantities are estimated, equation 14 becomes a tridiagonal system, which can be solved efficiently regardless of how many segments are set along the raypath (Wang and Houseman, 1994; Wang and Pratt, 2000). This is obviously a great advantage over the previous scheme which involves calculation of the Jacobian matrix and its inverse. Matrix inverse is an expensive calculation, proportional exponentially to the number of segments along the raypath.
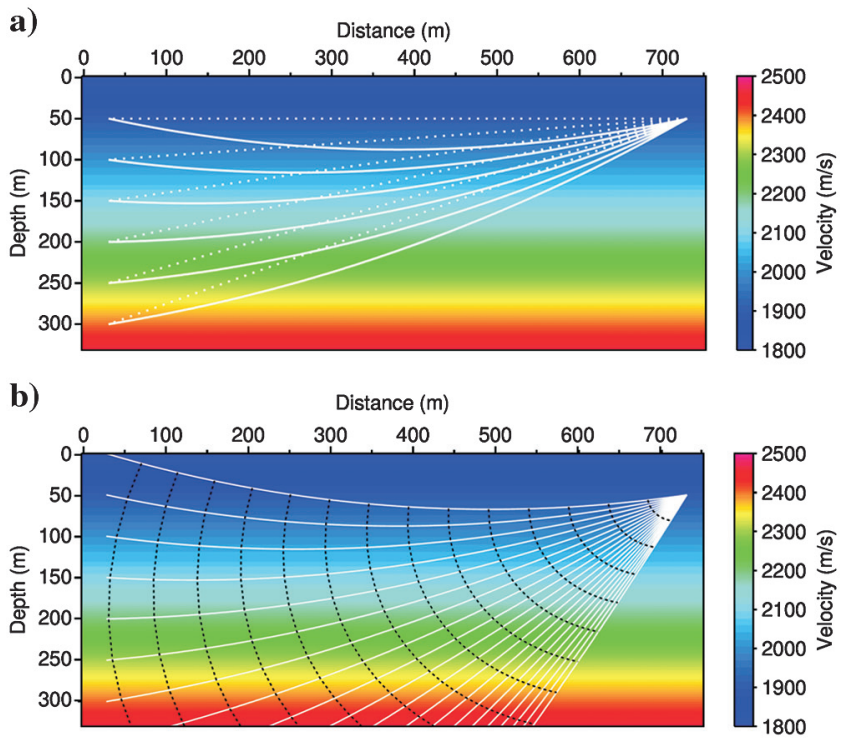

c)

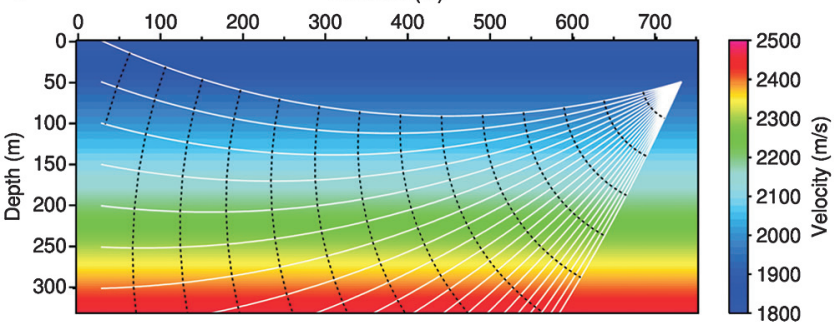

Figure 2. (a) Raypaths are initially set as dashed straight lines and the ray-tracing procedure converges steadily toward solid curves. (b) In anisotropic media with $\gamma_{c}=1.5$, raypaths are not perpendicular to the wavefronts. (c) Raypaths in isotropic media with $\gamma_{\mathrm{c}}=1$ are normal to the wavefronts.
Solving the tridiagonal system produces an estimate $\tilde{\mathbf{w}}$, which is in $\left\{x_{j}, z_{j}\right\}$ and not their perturbations. Difference $\Delta \mathbf{w}=\tilde{\mathbf{w}}-\mathbf{w}^{(k-1)}$ between the current estimate $\tilde{\mathbf{w}}$ and the previous solution $\mathbf{w}^{(k-1)}$ is equivalent to perturbation $\Delta \mathbf{w}=[\mathbf{J}(\mathbf{w})]^{-1} \mathbf{f}(\mathbf{w})$ in Newton's formula 3. Therefore, the modified solution update should be in the form of

$$
\mathbf{w}^{(k)}=\mathbf{w}^{(k-1)} \pm c^{\beta}\left(\tilde{\mathbf{w}}-\mathbf{w}^{(k-1)}\right) .
$$

The step length $\pm c^{\beta}$ is determined again based on Fermat's minimum-time constraint.

\section{RAY TRACING IN ANISOTROPIC MODELS}

In both ray-tracing schemes, the anisotropic velocity $V(x, z, \theta)$ is angle-dependent, where the wave propagation angle $\theta$ is measured against the vertical axis. It can be in any form as long as this directional velocity $V(x, z, \theta)$ along a raypath can be estimated numerically (Backus, 1962; Gassmann, 1964; Thomsen, 1986; Alkhalifah, 1998; Fomel, 2004). The examples presented in this paper assume the anisotropic velocity in a simple elliptical form

$$
V(\theta)=V_{\mathrm{h}} \sin ^{2} \theta+V_{\mathrm{v}} \cos ^{2} \theta,
$$

where $V_{\mathrm{v}}$ and $V_{\mathrm{h}}$ are the maximum vertical and horizontal velocities, respectively. A single anisotropy parameter may be defined by $\gamma=V_{\mathrm{h}} / V_{\mathrm{v}}$, the ratio of the major axis to the minor axis of the ellipse (Wang, 2011). Both $V_{\mathrm{h}}(x, z)$ and $V_{\mathrm{v}}(x, z)$, and in turn the parameter $\gamma(x, z)$ are functions of space.

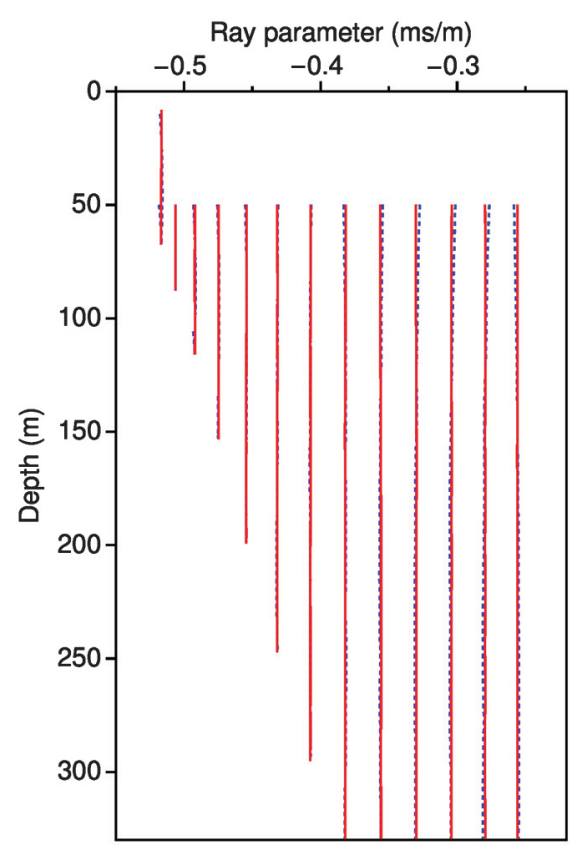

Figure 3. Dotted blue curves and solid red curves are ray parameter values of raypaths obtained from scheme 1 and scheme 2 , respectively. In the anisotropic media, the ray parameter at any point of a raypath should be a constant theoretically. The expectations of ray parameters for 13 curves are $-0.517,-0.506,-0.492$, $-0.475,-0.454,-0.432,-0.407,-0.382,-0.356,-0.330$, $-0.304,-0.280$, and $-0.256 \mathrm{~ms} / \mathrm{m}$. 
Figure $2 \mathrm{a}$ is a simple velocity model in which the maximum horizontal velocity is in $1 \mathrm{D}, V_{\mathrm{h}}(z)$, with a vertical gradient of $2 \mathrm{~m} / \mathrm{s}$ per meter, and the anisotropy parameter $\gamma(x, z)$ is constant, $\gamma_{\mathrm{c}}=1.5$. In numerical calculation, an initial raypath is a dashed straight line, and ray tracing converges steadily to a solid curve, thanks to the neat solution to a nonlinear system. Almost identical raypaths are produced by the two schemes, even one of which is an approximate expression for the raypath equations.

In anisotropic media, the raypaths are not perpendicular to the wavefronts (dotted black curves), as shown in Figure 2b. In contrast, rays in isotropic media with $\gamma_{\mathrm{c}}=1$ are normal to the wavefronts (dotted black curves), as shown in Figure 2c. The accuracy of the energetic raypaths in Figure $2 \mathrm{~b}$ will be further verified by the following analytical solution.

Snell's law holds in either anisotropic or isotropic media; that is, the ray parameter is constant along a raypath following the energy flux. The ray parameter is generally taken as horizontal phase slowness, $p_{x}=\sin \phi / v(z, \phi)$, defined by the phase angle and the phase velocity. For the vertically variable $1 \mathrm{D}$ anisotropic velocity model, $V(x, z, \theta)=V(z, \theta)$, the ray parameter can be expressed analytically in terms of the ray angle and the group velocity along a raypath (Slawinski and Webster, 1999), as follows

$$
p_{x}=\frac{\sin \theta}{V(z, \theta)}+\cos \theta \frac{\partial}{\partial \theta}\left(\frac{1}{V(z, \theta)}\right) .
$$

The second term on the right-hand side is the contribution of the directional velocity, as it will vanish when $V(z, \theta)=V(z)$. With the directional velocity defined as 16 , the ray parameter is given by

$$
p_{x}=\frac{\sin \theta}{V(z, \theta)}\left(3-2 \frac{V_{\mathrm{h}}(z)}{V(z, \theta)}\right) .
$$

For each raypath in anisotropic media (Figure $2 b$ ), the corresponding ray parameter calculated by equation 18 is a constant, as it appears as a straight line in Figure 3. Different vertical lines correspond to 13 raypaths, for which the source is fixed at $(730,50) \mathrm{m}$, and receivers are at depth $z=0,50,100, \ldots$, with $50 \mathrm{~m}$ interval, and distance $x=30 \mathrm{~m}$. The expectations (arithmetic means) of 13 ray parameter curves are: $-0.517,-0.506,-0.492,-0.475,-0.454$, $-0.432, \quad-0.407, \quad-0.382, \quad-0.356, \quad-0.330$, $-0.304,-0.280$, and $-0.256 \mathrm{~ms} / \mathrm{m}$.

A careful comparison in Figure 3 indicates that scheme 2 is slightly more accurate than scheme 1 , as the resultant ray parameters of scheme 2 (in red) are straighter than those of scheme 1 (dotted blue curves). For scheme 1, the standard deviations of 13 ray parameter curves are: 0.566 , $0.053,0.468, \quad 0.322, \quad 0.261,0.241,0.211$, $0.453,0.593,0.933,1.003,1.121,0.887\left(\times 10^{-3} \mathrm{~ms} / \mathrm{m}\right)$. For scheme 2, the standard deviations are: $0.093,0.032,0.026$, $0.063,0.082,0.096,0.081,0.160,0.169,0.182,0.146,0.102$, $0.100\left(\times 10^{-3} \mathrm{~ms} / \mathrm{m}\right)$. The overall averages of deviations in schemes 1 and 2 are $0.547 \times 10^{-3}$ and $0.102 \times 10^{-3} \mathrm{~ms} / \mathrm{m}$, respectively.

For a complicated velocity model, there will be some discrepancies. Figure 4 is an actual anisotropic velocity model, $V_{\mathrm{h}}(x, z)$ and $\gamma(x, z)$, reconstructed from seismic tomography of a field crosshole seismic data set (Rao and Wang, 2011). For ray tracing in such com-
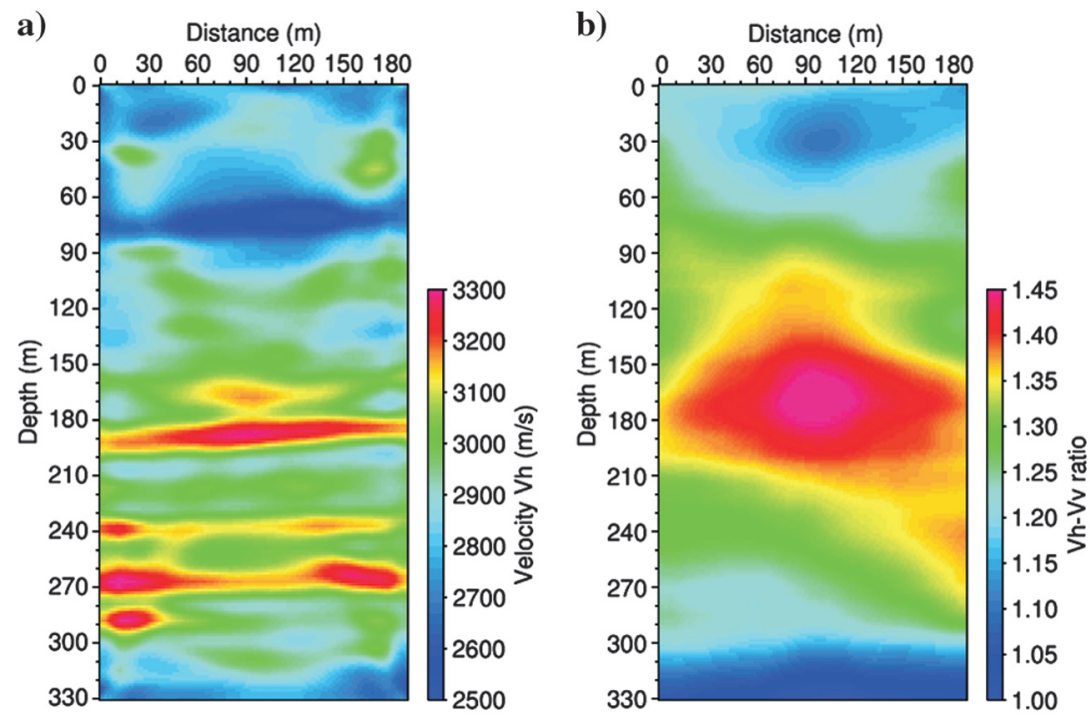

Figure 4. An actual anisotropic velocity model obtained from seismic tomography of a field crosshole seismic data set. (a) The horizontal velocity model, $V_{\mathrm{h}}(x, z)$. (b) The anisotropy parameter model, $\gamma(x, z)$.

a)

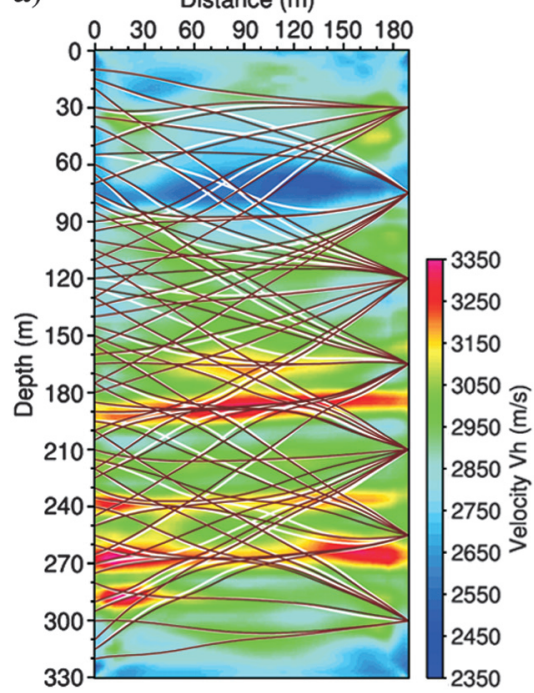

b)

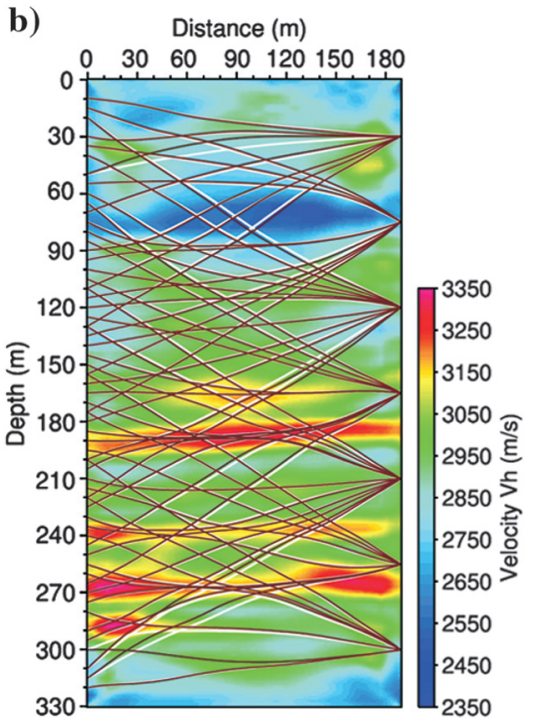

Figure 5. (a) Raypaths (brown) in the anisotropic velocity model with variable $\gamma(x, z)$ function, in comparison with raypaths (white) in an isotropic velocity model with $\gamma_{c}=1$ (i.e., $V_{\mathrm{v}}=V_{\mathrm{h}}$ ). (b) Raypaths (brown) in the actual anisotropic velocity model with variable $\gamma(x, z)$ function, in comparison with raypaths (white) in an anisotropic velocity model with $\gamma_{\mathrm{c}}=1.5$. 
plicated anisotropic media, when using the minimum-time constraint in step length $\left( \pm c^{\beta}\right)$ selection, a low time limit is also needed, to ensure that the update is not too far away from the current position and thus to secure a steady convergence for such a high nonlinear problem. It is set as $T \geq(1-\mu) T^{(k-1)}$, where $T^{(k-1)}$ is the traveltime obtained from the previous iteration, and $\mu \leq 0.2$ is set in this case. In addition, two unknown variable groups $\left\{z_{j}\right\}$ and $\left\{x_{j}\right\}$ are solved consecutively within each iteration, instead of a simultaneous solution, to further stabilize the procedure. a)

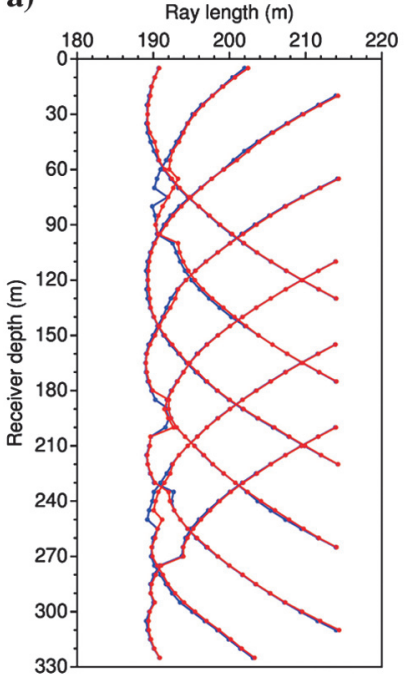

b)

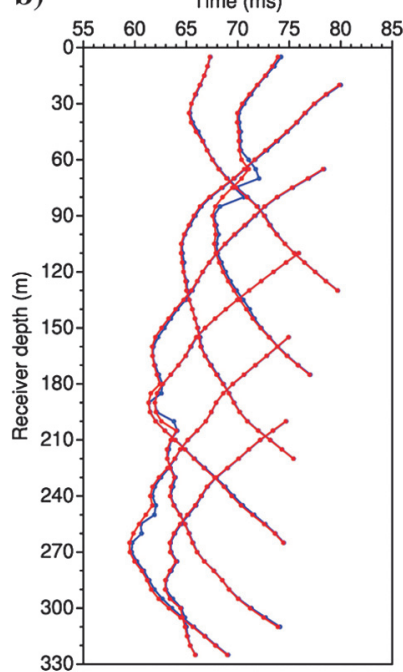

Figure 6. Ray lengths and traveltimes through the anisotropic velocity model with variable $\gamma(x, z)$ function, obtained from scheme 1 (in blue) and scheme 2 (in red). Source positions can be found from Figure 5 (the right-hand side of each panel).

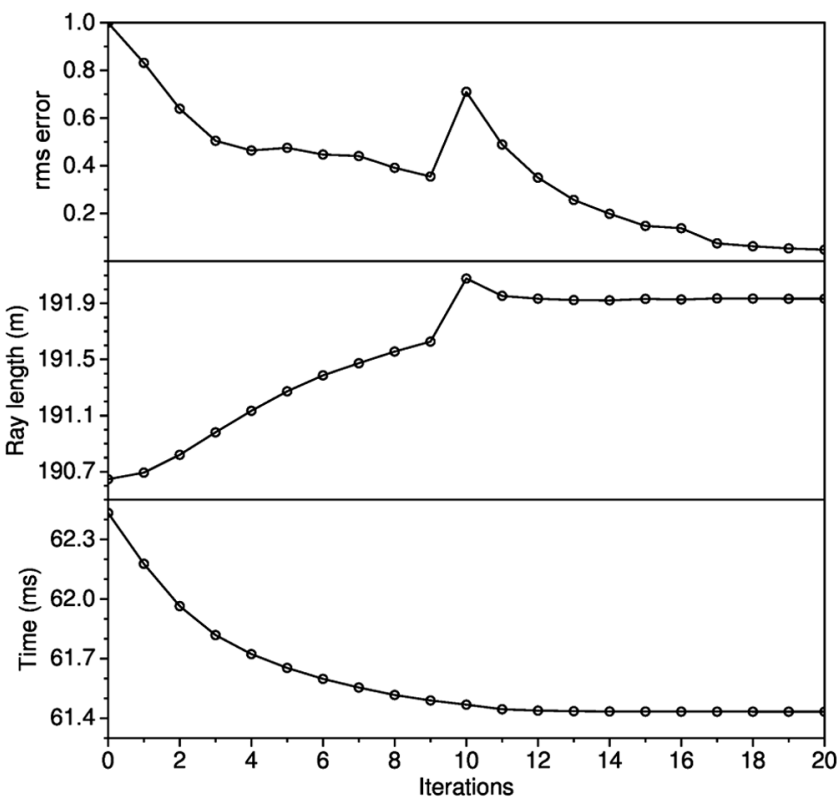

Figure 7. The convergence, measured by the normalized rms error of the system, the ray length, and the travel time along a raypath. It uses scheme 2 by solving a tridiagonal system.
Results in Figure 5a show that raypaths (brown) in the anisotropic medium are generally straightener than raypaths (white) in the isotropic medium with $\gamma_{\mathrm{c}}=1$. In the isotropic medium, raypaths are controlled purely by velocity variations. Because of the additional influence of $\gamma(x, z)$ function, raypaths are less curved in the anisotropic medium (for $\gamma>1$ ). The same observation can also be made in Figure 5b, in which raypaths (white) in the anisotropic medium with constant $\gamma_{\mathrm{c}}=1.5$ are even straightener than raypaths (brown) in the actual anisotropic medium with $2 \mathrm{D}$ variable $\gamma(x, z)$.

This comparison may be good evidence for the viability of a linear perturbation method that approximates sought raypaths in the anisotropic media based on known raypaths in the isotropic media. However, the nonlinear solution method proposed here with Fermat's minimum-time constraint is capable of finding raypaths in isotropic and anisotropic media, even with a simple initial guess such as a straight line.

Figure 6 compares the results of scheme 1 (in blue) and scheme 2 (in red). The comparison suggests that scheme 2 is more reliable than scheme 1, for the complicated model case. Wherever there is any discrepancy, scheme 2 always produces a longer path but a shorter traveltime than scheme 1 does.

Recall that the solution does not minimize the error of the system in each iteration, not even for the tridiagonal system in scheme 2 . Instead it attempts to find one associated with minimal traveltime. Figure 7 displays convergence curves measured by the (normalized) root-mean-square (rms) error of the system, the total length and the traveltime $T$ along the raypath, for ray tracing between an arbitrarily selected source-receiver pair, with source at $(189,165) \mathrm{m}$ and receiver at $(0,190) \mathrm{m}$. There are some oscillations which appeared on the rms error curve and the ray length curve which have a decreasing and increasing trend, respectively. However, as shown in Figure 7, the traveltime curve decreases steadily and monotonically through iterations, even in an iteration where the length has a big jump.

\section{CONCLUSIONS}

This paper proposes a modification to the conventional Newtontype iterative algorithm for solving the highly nonlinear problem of ray tracing in anisotropic media. It incorporates a physical principle with an algebraic problem, by enforcing Fermat's minimum-time principle as a constraint for the solution update to stabilize the convergence of a highly nonlinear problem.

This paper presents two bending schemes for ray tracing in anisotropic media. Both are expressed in terms of the group velocity and the ray angle. The first scheme involves newly derived raypath equations. These raypath equations are approximate for the anisotropic case but because they are constrained by Fermat's minimumtime principle, ray tracing steadily converges to the true solution.

The second scheme based on the minimum variation principle is more efficient than the first one, as it solves a tridiagonal system and does not need to compute the Jacobian matrix and its inverse in the iteration. However, rather than directly using the solution obtained from the error minimization in the system, the solution update is also constrained by Fermat's minimum-time principle because of the high nonlinearity of the problem for seismic ray tracing in anisotropic media.

The generalization for ray tracing through a 3D model would be a fairly straightforward case. However, further development is needed 
for general 3D anisotropic media addressing model parameterization and velocity definition issues.

\section{ACKNOWLEDGMENTS}

The author is grateful to the sponsors of the Centre for Reservoir Geophysics, Imperial College London, for supporting this research.

\section{REFERENCES}

Alkhalifah, T., 1998, Acoustic approximations for processing in transversely isotropic media: Geophysics, 63, 623-631, doi: 10.1190/1.1444361.

Backus, G. E., 1962, Long-wave elastic anisotropy produced by horizontal layering: Journal of Geophysical Research, 67, 4427-4440, doi: 10.1029/ JZ067i011p04427.

Červený, V., 2001, Seismic ray theory: Cambridge University Press.

Červený, V., 2002, Fermat's variational principle for anisotropic inhomogeneous media: Studia Geophysica et Geodaetica, 46, 567-588, doi: 10.1023/A:1019599204028.

Červený, V., and J. Jech, 1982, Linearized solutions of kinematic problems of seismic body waves in inhomogeneous slightly anisotropic media: Journal of Geophysics, 51, 96-104.

Červený, V., and I. Pšenčík, 2005, Plane waves in viscoelastic anisotropic media - Part I, theory: Geophysical Journal International, 161, 197-212, doi: 10.1111/j.1365-246X.2005.02589.x.

Crampin, S., 1981, A review of wave motion in anisotropic and cracked elastic-media: Wave Motion, 3, 343-391, doi: 10.1016/0165-2125(81) 90026-3.

Farra, V., and R. Madariaga, 1987, Seismic waveform modelling in heterogeneous media by ray perturbation theory: Journal of Geophysical Research, 92, 2697-2712, doi: 10.1029/JB092iB03p02697.

Fomel, S., 2004, On anelliptic approximations for qP velocities in VTI media: Geophysical Prospecting, 52, 247-259, doi: 10.1111/j.1365-2478 .2004.00413.x

Fryer, G. J., and L. N. Frazer, 1984, Seismic waves in stratified anisotropic media: Geophysical Journal of the Royal Astronomical Society, 78, 691710, doi: 10.1111/j.1365-246X.1984.tb05065.x.

Gassmann, F., 1964, Introduction to seismic travel time methods in anisotropic media: Pure and Applied Geophysics, 58, 63-112, doi: 10.1007/ BF00879140.
Hanyga, A., 1982, The kinematic inverse problem for weakly laterally inhomogeneous anisotropic media: Tectonophysics, 90, 253-262, doi: 10 $.1016 / 0040-1951(82) 90266-9$.

Jech, J., and I. Pšenčík, 1989, First-order perturbation method for anisotropic media: Geophysical Journal International, 99, 369-376, doi: 10.1111/j .1365-246X.1989.tb01694.x.

Julian, B. R., and D. Gubblins, 1977, Three-dimensional seismic ray tracing: Journal of Geophysics, 43, 95-113.

Kelley, C. T., 2003, Solving nonlinear equations with Newton's method: SIAM.

Pereyra, V., W. H. K. Lee, and H. B. Keller, 1980, Solving two-point seismic-ray tracing problems in a heterogeneous medium, Part 1, A general adaptive finite difference method: Bulletin of the Seismological Society of America, 70, 79-99.

Rao, Y., and Y. Wang, 2011, Crosshole seismic tomography including the anisotropy effect: Journal of Geophysics and Engineering, 8, 316-321, doi: 10.1088/1742-2132/8/2/016.

Rawlinson, N., J. Hauser, and M. Sambridge, 2007, Seismic ray tracing and wavefront tracking in laterally heterogeneous media: Advances in Geophysics, 49, 203-273, doi: 10.1016/S0065-2687(07)49003-3.

Shearer, P. M., and C. H. Chapman, 1988, Ray tracing in anisotropic media with a linear gradient: Geophysical Journal, 94, 575-580, doi: 10.1111/j 1365-246X.1988.tb02277.x.

Slawinski, M. A., and P. S. Webster, 1999, On generalized ray parameters for vertically inhomogeneous and anisotropic media: Canadian Journal of Exploration Geophysics, 35, 28-31.

Thomsen, L., 1986, Weak elastic anisotropy: Geophysics, 51, 1954-1966, doi: 10.1190/1.1442051.

Vavryčuk, V., 2006, Calculation of the slowness vector from the ray vector in anisotropic media: Proceedings of the Royal Society of London, Series A, Mathematical and Physical Science, 462, 883-896, doi: 10.1098/rspa .2005 .1605 .

Vavryčuk, V., 2008, Real ray tracing in anisotropic viscoelastic media: Geophysical Journal International, 175, 617-626, doi: 10.1111/j.1365-246X .2008.03898.x.

Wang, Y., 2011, Seismic anisotropy estimated from P-wave arrival times in crosshole measurements: Geophysical Journal International, 184, 1311-1316, doi: 10.1111/j.1365-246X.2010.04907.x.

Wang, Y., 2013, Simultaneous computation of seismic slowness paths and the traveltime field in anisotropic media: Geophysical Journal International, 195, no. 2, 1141-1148, doi: 10.1093/gji/ggt 278 .

Wang, Y., and G. A. Houseman, 1994, Inversion of reflection seismic amplitude data for interface geometry: Geophysical Journal International, 117, 92-110, doi: 10.1111/j.1365-246X.1994.tb03305.x.

Wang, Y., and R. G. Pratt, 2000, Seismic amplitude inversion for interface geometry of multi-layered structures: Pure and Applied Geophysics, 157, 1601-1620, doi: 10.1007/PL00001052. 